\title{
A novel predictor of infarct-related artery patency before percutaneous intervention and in-hospital outcomes for ST-segment elevation myocardial infarction patients: serum bilirubin level
}

\author{
Halit Acet, Faruk Ertaş, Mehmet Ata Akıl, Nihat Polat, Mesut Aydın, Abdurrahman Akyüz, Hilal Ayçiçek, \\ Sait Alan \\ Department of Cardiology, Faculty of Medicine, Dicle University, Diyarbakır, Turkey
}

Postep Kardiol Inter 2014; 10, 2 (36): 91-97 DOI: $10.5114 /$ pwki.2014.43513

\begin{abstract}
A bstract
Introduction: Previous studies have reported a relationship between serum bilirubin levels and coronary artery disease (CAD). However, data are rare up to now regarding the relation of bilirubin levels with infarct-related artery (IRA) patency in the setting of ST-segment elevation myocardial infarction (STEMI). Moreover, previous studies reported that increased bilirubin was related to impaired post-intervention coronary flow. To our knowledge, the association between serum total bilirubin (TB) levels and pre-primary percutaneous coronary intervention (PCI) with patency of IRA flow in STEMI patients has not been investigated.

Aim: To evaluate the association of TB with pre-primary PCI, coronary flow and in-hospital major adverse cardiac events (MACE) in patients with STEMI.

Material and methods: A total of 360 consecutive patients with STEMI (mean age $=61.4 \pm 13.7$ years) admitted within $12 \mathrm{~h}$ from the time of symptom onset were enrolled. Patients were divided into 2 groups based on the serum TB levels. We defined normal flow as pre-PCI TIMI 3 flow, while impaired flow was defined as pre-PCI TIMI $\leq 2$ flow.

Results: Pre-PCI impaired flow was higher in the TB group than pre-PCI normal flow $(p<0.001)$. In-hospital mortality and MACE were significantly higher in the high TB group $(p=0.002, p<0.001$ respectively). In the receiver operating characteristic curve analysis, TB $>0.825 \mathrm{mg} / \mathrm{dl}$ predicted impaired IRA flow before $\mathrm{p}-\mathrm{PCl}$ with a sensitivity of $79 \%$ and specificity of $71 \%$.

Conclusions: The TB is an inexpensive and readily available marker for STEMI patients undergoing PCI. It can be used for risk stratification in this patient population.
\end{abstract}

Key words: bilirubin, percutaneous coronary intervention, novel predictor.

\section{Introduction}

The basal thrombolysis in myocardial infarction (TIMI) flow in an infarct-related artery (IRA) is crucial for patients with ST-segment elevation myocardial infarction (STEMI) undergoing primary percutaneous coronary intervention $(\mathrm{PCI})$. However, pre-PCI patency of IRA in patients with STEMI is a major determinant of post-PCI TIMI flow; this is related to improved clinical outcome [1]. Early and rapid restoration of coronary flow in an IRA improves ventricular performance, lowers the incidence of congestive heart failure, and decreases mortality compared with patients without initial TIMI flow 3 in patients with STEMI [2].
Bilirubin is a bile pigment formed as the end product of the breakdown of heme [3]. The heme oxygenase ( $\mathrm{HO})$ enzyme that is responsible for the degradation of heme groups in the metabolism of bilirubin is a stress-inducible enzyme and has antioxidative features [4]. There are 2 isozymes of this enzyme, $\mathrm{HO}-1$ and $\mathrm{HO}-2$ [5]. The HO- 1 is induced by various cellular stresses but is not expressed under normal conditions. A large number of outcomes are possible [1]. Previous studies demonstrated that $\mathrm{HO}-1$ can be induced by stress, including as part of the acute phase response [5]. It has been demonstrated that $\mathrm{HO}-1$ enzyme activity is markedly upregulated in the myocardium in response to infarction [6].

\section{Corresponding author:}

Halit Acet MD, Assist. Prof., Department of Cardiology, Faculty of Medicine, Dicle University, 21090 Diyarbakır, Turkey, phone: +90 5053190183, e-mail: halitacet@gmail.com

Received: 3.01.2014, accepted: 20.01.2014. 
Previous studies have reported a relationship between serum bilirubin levels and the risk of coronary artery disease (CAD) [7]. However, data are rare up to now regarding the relation of bilirubin levels with IRA patency in the setting of STEMI. Moreover, previous studies reported that increased bilirubin was related to impaired post-intervention coronary blood flow in patients with STEMI [8]. To our knowledge, the relationship between serum total bilirubin (TB) levels and pre$\mathrm{PCl}$ with patency of IRA flow in STEMI patients has not been investigated.

\section{Aim}

Therefore, in this study, we evaluated the relationship between TB IRA flow as confirmed by angiography before receiving $\mathrm{PCl}$ and in-hospital major adverse cardiac events (MACE) in patients with STEMI.

\section{Material and methods}

\section{Study population}

A total 760 consecutive patients with STEMI undergoing $\mathrm{PCl}$ within $12 \mathrm{~h}$ from the time of symptom onset between January 2012 and December 2013 were included in this study. The STEMI was defined based on the criteria formulated by the American College of Cardiology and the European Society of Cardiology [9] as an increase in troponin I > $1 \mathrm{ng} / \mathrm{ml}$ with a new ST elevation measured from the J point in $\geq 2$ contiguous leads with at least $0.2 \mathrm{mV}$ in leads $\mathrm{V} 1, \mathrm{~V} 2$ and $\mathrm{V} 3$ or at least $0.1 \mathrm{mV}$ in the remaining leads during the first $12 \mathrm{~h}$ after the onset of symptoms.

Patients with severe liver disease, autoimmune disease, known malignancy, hematological disorders, severe valvular disease, known cases of hypothyroidism, inflammatory or infectious diseases, a history of bleeding diathesis, patients not given a $\mathrm{PCl}$, a bilirubin value was missing or unavailable and follow-up was not documented before $\mathrm{PCl}$, patients who were treated with thrombolytic therapy or the use of glycoprotein IIb/IIla inhibitors, patients with poor echocardiographic windows, and patients who presented $>12 \mathrm{~h}$ from the onset of symptoms were excluded from the study. After these exclusions were accounted for, the final study population consisted of 360 patients. All patients underwent a complete physical examination; coronary risk factors, and their medical histories and clinical features were recorded.

In-hospital follow-up and MACE were defined as acute stent thrombosis, cardiogenic shock, new advanced heart failure, pulmonary edema, complete atrioventricular block (AVB) requiring a transient pacemaker, serious ventricular arrhythmia, major bleeding requiring blood transfusion and in-hospital mortality during the in-hospital follow-up period. In-hospital mortality had to be verified as death due to myocardial infarction, cardiac arrest or other cardiac causes. Acute in-stent thrombosis was defined as an abrupt onset of cardiac symptoms after stent deployment in the first $24 \mathrm{~h}$ and angiographic evidence of a flow-limiting thrombus near a previously placed stent. Cardiogenic shock was defined as marked and persistent (> $30 \mathrm{~min}$ ) hypotension with systolic arterial pressure $<80 \mathrm{~mm} \mathrm{Hg}$ and signs of hypoperfusion due to left ventricular dysfunction, right ventricular infarction, or mechanical complications. New advanced heart failure was defined as New York Heart Association functional classification $\geq$ III. Serious ventricular arrhythmia was defined on admission as advanced ventricular fibrillation-tachycardia or asystole $48 \mathrm{~h}$ later. Patients were also evaluated according to the Killip clinical examination classification [10].

\section{Blood samples and echocardiography}

Venous blood samples were drawn when the patient initially presented to the emergency room or the intensive coronary care unit (ICCU) before PCI. Hematologic indexes counts were measured by an automated hematology analyzer (Abbott Cell-Dyn 3700; Abbott Laboratory, Abbott Park, Illinois). Bilirubin and other biochemical parameters were measured using the Abbott Architect C16000 auto analyzer (Abbott Laboratory). Normal total bilirubin and direct bilirubin levels were defined according to our laboratory $(0.2-1 \mathrm{mg} / \mathrm{dl}$ and $0.0-0.5 \mathrm{mg} / \mathrm{dl}$ respectively). Transthoracic two-dimensional echocardiography was performed on ICCU admission in order to measure left ventricular ejection fraction (LVEF) (Vivid S6, GE Medical Systems, USA).

\section{Coronary angiography (TIMI flow)}

All patients underwent selective coronary angiography using the Judkins technique. The $\mathrm{PCl}$ procedures were performed with a standard femoral approach using a $7 \mathrm{Fr}$ guiding catheter. Digital angiograms were analyzed by three independent and experienced interventional cardiologists, blinded to all data. Coronary blood flow patterns before $\mathrm{PCl}$ were subjected to a thorough evaluation on the basis of TIMI flow grade, using grades $0,1,2$, and 3 [11]. We defined normal flow or spontaneous coronary reperfusion (SR) as pre-PCI TIMI 3 flow (SR group), while impaired or non-SR flow was defined as pre-PCI TIMI $\leq 2$ flow (non-SR group). For all study participants, only one artery was identified as the IRA. Coronary vessel disease was defined as a greater than $50 \%$ stenosis in one of the major coronary arteries. The Gensini scoring system was used to determine the severity of CAD $[12,13]$. TIMI flow grade was determined by three independent interventional cardiologists. Intra- and inter-observer reliability was determined from a random sample of 100 patients from the study sample. 


\section{Statistical analysis}

All analyses were performed using SPSS for Windows version 18.0 (SPSS Inc. Chicago, Illinois, USA). Continuous variables were expressed as mean \pm standard deviation (SD) and categorical variables were expressed as percentages. Distribution of continuous variables was assessed with a two-sample Kolmogorov-Smirnov test. Comparisons of categorical and continuous variables between the two groups (high and low TB level) were performed using the $\chi^{2}$ or Fisher's exact test and independent samples $t$ or Mann-Whitney $U$ test, respectively. Relationships between the variables were examined with Spearman's correlation coefficients. The inter-rater agreement between the three cardiologists in determining intra- and inter-observer agreement for the coronary blood flow patterns before $\mathrm{PCl}$ were subjected to a thorough evaluation on the basis of TIMI flow, investigated using the Kappa (к) test. Statistical significance was defined as $p<0.05$. The cut-off values for TB for predicting non-SR, in-hospital MACE and in-hospital death with corresponding sensitivity and specificity were estimated by receiver operator characteristic (ROC) curve analysis.

The study protocol was in accordance with the Declaration of Helsinki and was approved by the local Ethics Committee.

\section{Results}

A total of 360 patients were included in the data analysis. 101 (28\%) were in the SR group and 259 (72\%) were in the non-SR group. Out of all the study participants, 130 (36\%) were in the low TB group and the remaining 230 (64\%) were stratified into the high TB group. The baseline characteristics of the 2 groups are summarized in Table I. Killip classes III-IV were more common in high TB group patients ( $p=0.017$ ).

Initial laboratory findings were compared according to the high TB group and the low TB group (Table II). In the high TB group, TB $(p<0.001)$, direct bilirubin $(p<0.001)$, aspartate amino transferase $(p=0.015)$, alanine amino transferase $(p=0.002)$, lactate dehydrogenase $(p=0.002)$, C-reactive protein (CRP) $(p=0.002)$, serum uric acid (UA) $(p<0.001)$, creatine $(p=0.017)$, peak troponin $(p<0.001)$, peak CK-MB $(p<0.001)$ and neutrophil to lymphocyte ratio (NLR) $(p=0.035)$ values were significantly greater than those of the low TB group. Moreover, mean platelet volume, hemoglobin, and serum glucose on admission were higher in the high TB group patients.

Angiographic characteristics are listed in Table III. Pre$\mathrm{PCI}$ TIMI flow 3 was higher in the low TB group than the high TB group ( $p<0.001)$. Similarly, the non-SR group TB $(p<0.001)$ value was significantly greater than that of the

Table I. Baseline characteristics of study patients

\begin{tabular}{|c|c|c|c|}
\hline Variables & $\begin{array}{l}\text { Low serum TB group } \\
\qquad(n=130)\end{array}$ & $\begin{array}{l}\text { High serum TB group } \\
\qquad(n=230)\end{array}$ & Value of $p$ \\
\hline Age, mean \pm SD [years] & $60.3 \pm 13.5$ & $62.0 \pm 13.8$ & 0.242 \\
\hline Sex, male, $n(\%)$ & $97(75)$ & $165(72)$ & 0.556 \\
\hline Hypertension, $n(\%)$ & $46(35)$ & $83(36)$ & 0.894 \\
\hline Diabetes mellitus, $n(\%)$ & $30(23)$ & $57(25)$ & 0.717 \\
\hline Smoking, $n(\%)$ & $74(57)$ & $127(55)$ & 0.754 \\
\hline Hyperlipidemia, $n(\%)$ & $8(6)$ & $9(4)$ & 0.336 \\
\hline Family history of CAD, $n(\%)$ & $32(25)$ & $39(17)$ & 0.079 \\
\hline Previous MI or CAD, $n(\%)$ & $8(6)$ & $10(4)$ & 0.450 \\
\hline Previous PCI, $n(\%)$ & $5(4)$ & $14(6)$ & 0.361 \\
\hline \multicolumn{4}{|l|}{ Killip class on admission, $n$ (\%): } \\
\hline$|-| \mid$ & $126(97)$ & $207(90)$ & \multirow{2}{*}{0.017} \\
\hline III-IV & $4(3)$ & $23(10)$ & \\
\hline Admission SBP, mean $\pm \mathrm{SD}[\mathrm{mm} \mathrm{Hg}]$ & $128.5 \pm 20.9$ & $127.4 \pm 25.2$ & $0.650^{*}$ \\
\hline Admission heart rate, mean \pm SD [bpm] & $82.6 \pm 15.6$ & $84.1 \pm 17.4$ & $0.416^{*}$ \\
\hline \multicolumn{4}{|l|}{ STEMI location, $n(\%)$ : } \\
\hline Anterior & $61(47)$ & $105(46)$ & \multirow{2}{*}{0.816} \\
\hline Nonanterior & $69(53)$ & $125(55)$ & \\
\hline Duration of chest pain, mean \pm SD [h] & $5.5 \pm 3.2$ & $5.9 \pm 3.5$ & $0.194^{*}$ \\
\hline Admission LVEF, mean \pm SD (\%) & $44.1 \pm 9.7$ & $42.8 \pm 10.9$ & $0.259^{*}$ \\
\hline
\end{tabular}


Table II. Initial laboratory findings of study patients

\begin{tabular}{|c|c|c|c|}
\hline Variables & $\begin{array}{l}\text { Low serum TB } \\
\quad(n=130)\end{array}$ & $\begin{array}{l}\text { High serum TB } \\
(n=230)\end{array}$ & Value of $p$ \\
\hline Total bilirubin [mg/dl] & $0.56 \pm 0.18$ & $1.38 \pm 0.32$ & $<0.001^{*}$ \\
\hline Direct bilirubin $[\mathrm{mg} / \mathrm{dl}]$ & $0.21 \pm 0.09$ & $0.33 \pm 0.32$ & $<0.001^{\star}$ \\
\hline Aspartate amino transferase & $89.53 \pm 87.2$ & $123.89 \pm 141.99$ & $0.015^{\star}$ \\
\hline Alanine amino transferase & $32.54 \pm 30.80$ & $54.65 \pm 90.34$ & $0.002^{\star}$ \\
\hline Lactate dehydrogenase & $334.96 \pm 171.59$ & $443.82 \pm 306.83$ & $0.002^{\star}$ \\
\hline C-reactive protein & $0.71 \pm 0.72$ & $2.33 \pm 4.95$ & 0.002 \\
\hline Uric acid [mg/dl] & $6.34 \pm 2.54$ & $7.31 \pm 2.60$ & $<0.001^{*}$ \\
\hline Peak troponin I [ng/ml] & $38.1 \pm 36.9$ & $64.7 \pm 34.7$ & $<0.001^{\star}$ \\
\hline Peak CK-MB mass [ng/ml] & $140.9 \pm 115.9$ & $207.8 \pm 106.4$ & $<0.001^{\star}$ \\
\hline Glucose on admission [mg/dl] & $173.02 \pm 98.40$ & $175.27 \pm 84.64$ & 0.819 \\
\hline Creatinine on admission $[\mathrm{mg} / \mathrm{dl}]$ & $0.91 \pm 0.58$ & $0.93 \pm 0.37$ & $0.017^{\star}$ \\
\hline Total cholesterol [mg/dl] & $178.90 \pm 43.25$ & $178.85 \pm 37.35$ & 0.992 \\
\hline Low-density lipoprotein [mg/dl] & $116.22 \pm 34.72$ & $114.54 \pm 30.55$ & 0.633 \\
\hline High-density lipoprotein [mg/dl] & $36.04 \pm 9.45$ & $34.67 \pm 9.88$ & 0.199 \\
\hline Triglycerides [mg/dl] & $147.23 \pm 88.50$ & $153.73 \pm 94.74$ & 0.523 \\
\hline White blood cell count $[\mathrm{K} / \mu \mathrm{l}]$ & $13.07 \pm 4.06$ & $13.99 \pm 6.09$ & 0.123 \\
\hline Neutrophil count, NULL & $10.13 \pm 3.84$ & $11.31 \pm 5.59$ & 0.019 \\
\hline Lymphocyte count, NULL & $2.05 \pm 1.27$ & $1.93 \pm 1.22$ & 0.382 \\
\hline Neutrophil to lymphocyte ratio & $6.45 \pm 4.07$ & $7.71 \pm 5.39$ & $0.035^{\star}$ \\
\hline Hemoglobin $[\mathrm{g} / \mathrm{dl}]$ & $13.82 \pm 1.81$ & $13.94 \pm 1.54$ & 0.532 \\
\hline Mean platelet volume & $8.04 \pm 1.21$ & $8.35 \pm 1.62$ & 0.057 \\
\hline
\end{tabular}

Values are mean $\pm S D,{ }^{*}$ Mann-Whitney $U$ test, Student's t-test

Table III. Angiographic characteristics of patients

\begin{tabular}{|c|c|c|c|}
\hline Variables & $\begin{array}{l}\text { Low serum } \\
\text { TB group } \\
(n=130)\end{array}$ & $\begin{array}{l}\text { High serum } \\
\text { TB group } \\
(n=230)\end{array}$ & Value of $p$ \\
\hline \multicolumn{4}{|l|}{ IRA: } \\
\hline Left anterior descending & $64(49)$ & $108(47)$ & \multirow{3}{*}{0.889} \\
\hline Right & $41(32)$ & $94(41)$ & \\
\hline Circumflex & $25(19)$ & $28(12)$ & \\
\hline \multicolumn{4}{|l|}{ Narrowed coronary vessels: } \\
\hline 1 vessel & $63(49)$ & $91(40)$ & \multirow{2}{*}{0.101} \\
\hline$>1$ vessel & $67(52)$ & $139(60)$ & \\
\hline \multicolumn{4}{|l|}{ Initial TIMI flow (pre-PCI): } \\
\hline $0-1$ & $50(39)$ & $184(80)$ & \multirow{3}{*}{$<0.001$} \\
\hline 2 & $9(7)$ & $16(7)$ & \\
\hline 3 & $71(55)$ & $30(13)$ & \\
\hline Gensini score & $52.76 \pm 29.76$ & $65.24 \pm 31.05$ & $<0.001$ \\
\hline
\end{tabular}

Values are mean \pm SD or $n(\%), \chi^{2}$ test; Student's t-test, IRA-infarct-related artery, TIMI-Thrombolysis In Myocardial Infarction grade, PCI - percutaneous coronary intervention

SR group (Figure $1 \mathrm{~A})$. The Gensini score was higher in the high TB group as compared to the low TB group $(p<0.001)$.

The in-hospital adverse outcomes are listed in Table IV. The in-hospital death rate was greater in the high TB group than in the low TB group (14\% vs. $4 \%, p=0.002)$. MACE were more frequent in the high TB group than in the low TB group ( $30 \%$ vs. $12 \%, p<0.001)$. Similarly, in-hospital cardiogenic shock $(p=0.005)$, complete 
Table IV. In-hospital cardiac events according to serum bilirubin level

\begin{tabular}{lccc} 
Variables & $\begin{array}{c}\text { Low serum } \\
\text { TB group }\end{array}$ & $\begin{array}{c}\text { High serum TB } \\
\text { group }\end{array}$ & Value of $p$ \\
\hline In-hospital MACE & $15(12)$ & $68(30)$ & $<0.001$ \\
\hline Advanced heart failure & $6(5)$ & $18(8)$ & 0.241 \\
\hline Advanced pulmonary edema & $4(3)$ & $14(6)$ & 0.208 \\
\hline Cardiogenic shock & $3(2)$ & $24(10)$ & 0.005 \\
\hline Acute stent thrombosis & $2(2)$ & $7(3)$ & 0.380 \\
\hline Complete AVB requiring transient pacemaker & $2(2)$ & $16(7)$ & 0.023 \\
\hline Serious ventricular arrhythmia & $4(3)$ & $22(10)$ & 0.022 \\
\hline Major bleeding requiring blood transfusion & $1(0.8)$ & $0(0)$ & 0.183 \\
\hline In-hospital death & $5(4)$ & $33(14)$ & 0.002 \\
\hline Cardiopulmonary resuscitations & $5(4)$ & $37(16)$ & 0.001 \\
\hline Cardiac arrest on admission & $3(2)$ & $24(10)$ & 0.005 \\
\hline Hospitalization [days] & $4.8 \pm 2.9$ & $5.5 \pm 5.3$ & $0.107^{*}$
\end{tabular}

Values are mean $\pm S D$ or $n(\%), \chi^{2}$ test, Student's t-test, *Mann-Whitney U test, MACE - major adverse cardiovascular event, AVB - atrioventricular block
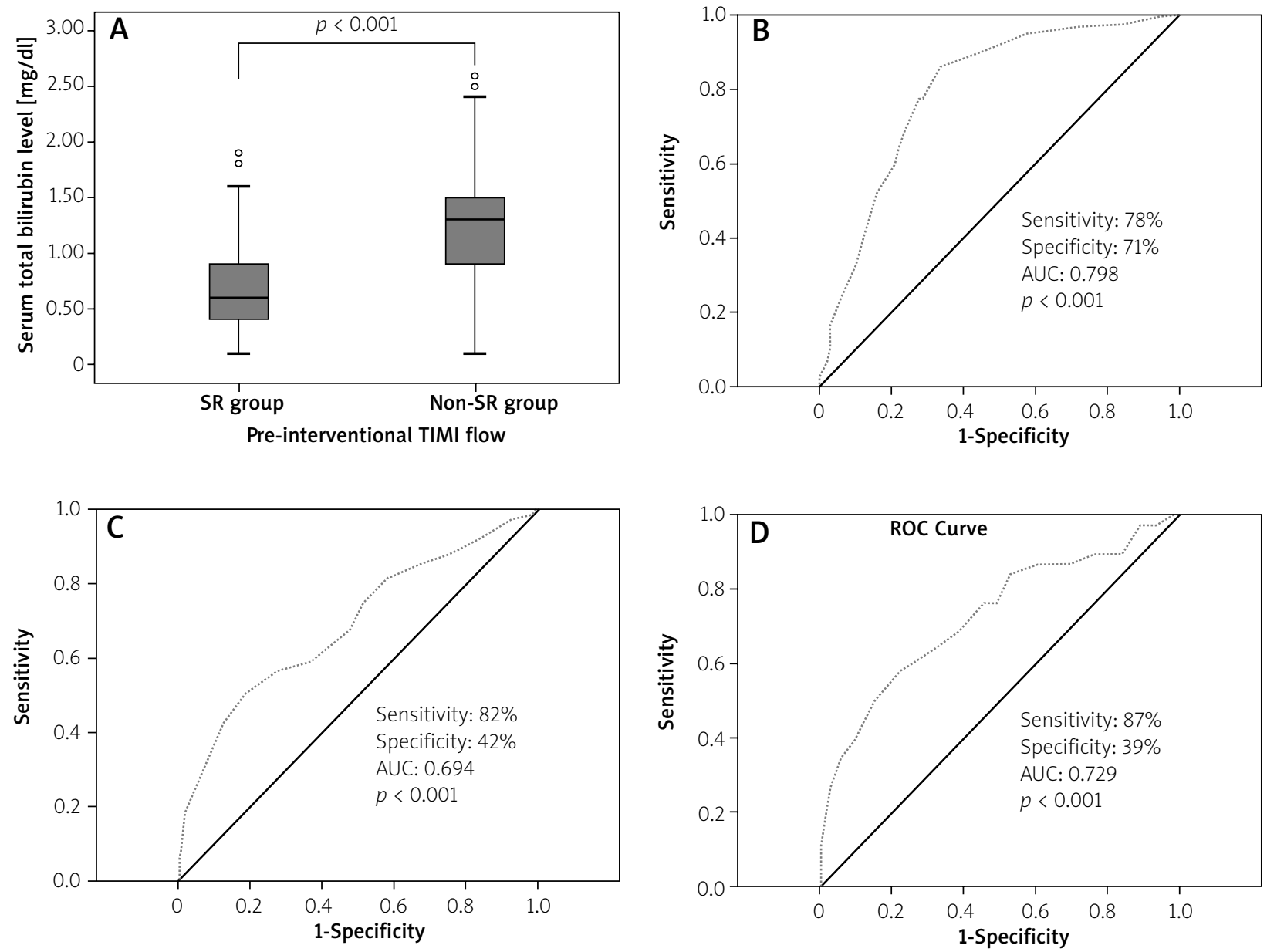

Figure 1. A - Box plot comparison of serum total bilirubin levels among SR and non-SR patient groups before primary $\mathrm{PCl}, \mathbf{B}$ - receiver operator characteristic curve analysis of serum total bilirubin in predicting pre-PCI IRA patency, $\mathbf{C}$ - receiver operator characteristic curve analysis of serum total bilirubin in predicting in-hospital major adverse outcomes, D - receiver operator characteristic curve analysis of serum total bilirubin in predicting in-hospital mortality 
AVB requiring a transient pacemaker $(p=0.023)$, severe ventricular arrhythmia $(p=0.022)$, cardiac arrest on admission $(p=0.005)$ and cardiopulmonary resuscitations ( $p=0.001$ ) were higher in the high TB group patients.

Spearman's correlation analysis revealed significant associations between a high TB value and UA $(r=0.199$, $p<0.001)$, NLR $(r=0.155, p=0.004)$, peak troponin $(r=0.128, p=0.015)$, peak CK-MB $(r=0.126, p=0.017)$ and Gensini score $(r=0.245, p<0.001)$.

A cut-off level of $0.825 \mathrm{mg} / \mathrm{dl}$ for TB predicted impaired IRA flow before $\mathrm{PCI}$ with a sensitivity of $77.6 \%$ and a specificity of $71.3 \%$ (ROC area under the curve (AUC) $0.798,95 \% \mathrm{Cl}: 0.745-0.853, p<0.001$ ) (Figure $1 \mathrm{~B}$ ). A cutoff level of $0.825 \mathrm{mg} / \mathrm{dl}$ for TB was identified as in STEMI for in-hospital MACE with a sensitivity of $81.9 \%$ and a specificity of $41.5 \%$ (ROC AUC $0.694,95 \% \mathrm{Cl}: 0.626-$ $0.763, p<0.001$ ) (Figure $1 \mathrm{C}$ ), and a TB value $>0.825 \mathrm{mg} / \mathrm{dl}$ was identified as an effective cut-off point in STEMI for in-hospital mortality with a sensitivity of $86.8 \%$ and a specificity of $38.8 \%$ (ROC AUC $0.729,95 \% \mathrm{Cl}: 0.635-$ $0.823, p<0.001$ ) (Figure $1 \mathrm{D}$ ).

The $\kappa$ value for intra-observer reliability was 0.907 $(p<0.001)$. The inter-observer reliability was a $\kappa$ of 0.889 $(p<0.001)$ before PCI TIMI flow grade assignments 0 to 3 . Inter-rater reliability and the intraclass correlation coefficient had an $r$-value of 0.961 with a $p<0.001$.

\section{Discussion}

Our data demonstrated that high serum TB was associated with impaired pre-PCI IRA flow and increased in-hospital MACE and death occurred in patients with STEMI. Also this study demonstrated increased TB levels that correlated with serum UA and NLR, which are markers of inflammation [14, 15]. In addition, we found that high levels of TB were significantly associated with increased CAD severity according to Gensini scoring results. Moreover, we found that TB values greater than $0.825 \mathrm{mg} / \mathrm{dl}$ predict initial IRA TIMI flow grades of less than 3, in-hospital MACEs and in-hospital death. The sensitivity and specificity for the association between TB and low IRA patency pre-PCI TIMI flow grades were $78 \%$ and $71 \%$, in hospital-MACE were $82 \%$ and $42 \%$, and in-hospital mortality was $87 \%$ and $39 \%$ respectively.

Bilirubin levels prolonged the survival of human ventricular myocytes against in situ generated oxyradicals [16]. It was also found that $\mathrm{HO}-1$ protein and $\mathrm{HO}$ activity are markedly upregulated in the myocardium in response to MI. The activation of HO-1 is an important defensive mechanism that may improve cytoprotection via its antioxidant activity, which may limit tissue damage and ameliorate the post-MI remodeling [17, 18]. A previous study tested the hypothesis that $\mathrm{HO}-1$ is activated in the myocardium after acute $\mathrm{MI}$ in humans [6]. In assessing the serum concentration of bilirubin levels and $\mathrm{HO}-1$ enzyme activity during the hospitalization period in pa- tients with acute $\mathrm{MI}$, it was found that HO-1 enzyme activity is a stress-inducible enzyme and closely correlated with serum bilirubin levels.

Gullu et al. [19] reported that greater serum bilirubin concentrations had a protective effect against impaired coronary flow reserve and coronary microvascular dysfunction. Erdogan et al. [20] evaluated undergoing coronary angiography for CAD and found that greater bilirubin levels were related to favorable coronary collateral flow in patients with chronic total occlusion. Okuhara et al. [6] reported that serum bilirubin levels were elevated in those with acute MI but not in nonacute MI. Celik et al. [8] found that serum bilirubin levels were independently associated with the development of post- $\mathrm{PCl}$ coronary no-reflow with TIMI flow grade as well as increased in-hospital MACE in patients with STEMI. Gul et al. [21] demonstrated that high TB was one of the independent predictors of in-hospital cardiovascular mortality and morbidity in patients with acute STEMI.

Wei et al. [22] found that serum TB levels correlated with the angiographic complexity of CAD. Erkan et al. [23] found a moderate and significant positive correlation between direct bilirubin levels and the Gensini score. There was no significant correlation between TB levels and the Gensini score. In contrast, we demonstrated that a high TB level is significantly associated with the Gensini score in patients with STEMI. In addition, Gensini score correlated with TB and direct bilirubin levels.

We demonstrated that serum TB levels, a sign of HO-1 enzyme activity, are significantly associated with $\mathrm{PCl}$ coronary flow and adverse outcomes during the in-hospital period. This suggests that the activity of the stress-inducible enzyme HO-1 is independently related to impaired pre-PCl coronary blood flow, in hospital-MACEs and death in patients with STEMI. These results could play an important role in better understanding the pathogenesis of oxidative stress associated diseases and may lead to the development of new treatment strategies in the management of patients with STEMI.

The present study has the well-known limitation of retrospective design and a small number of patients. In addition, it was a single-center study. However, our population contains homogeneous unselected patients with STEMI submitted to $\mathrm{PCl}$ within $12 \mathrm{~h}$ from the time of symptom onset, thus mirroring the real-world scenario. The other limitation is that we did not measure HO-1 activity, but we did examine NLR and UA markers of inflammation. Also there was a significant correlation between serum markers and serum TB level.

\section{Conclusions}

From the findings of the present study, we suggest that TB is an inexpensive and readily available biochemical marker for clinicians to use as a parameter and a powerful prognostic factor for patients undergoing PCI 
for STEMI. It can be used for risk stratification in this patient population.

\section{References}

1. Mehta RH, Harjai KJ, Cox D, et al. Clinical and angiographic correlates and outcomes of suboptimal coronary flow in patients with acute myocardial infarction undergoing primary percutaneous coronary intervention. J Am Coll Cardiol 2003; 42: 1739-61.

2. Stone GW, Cox D, Garcia E, et al. Normal flow (TIMI-3) before mechanical reperfusion therapy is an independent determinant of survival in acute myocardial infarction: analysis from the primary angioplasty in myocardial infarction trials. Circulation 2001 104: 636-41.

3. Stocker R, Yamamoto Y, McDonagh AF, et al. Bilirubin is an antioxidant of possible physiological importance. Science 1987; 235 1043-6.

4. Sahin O, Akpek M, Elcik D, et al. Bilirubin levels and the burden of coronary atherosclerosis in patients with STEMI. Angiology 2013; 64: 200-4.

5. Breimer LH, Mikhailidis DP. Could carbon monoxide and bilirubin be friends as well as foes of the body? Scand I Clin Lab Invest 2010; 70: 1-5.

6. Okuhara K, Kisaka T, Ozono R, et al. Change in bilirubin level following acute myocardial infarction is an index for heme oxygenase activation. South Med J 2010; 103: 876-81.

7. Schwertner HA, Fischer JR Jr. Comparison of various lipid, lipoprotein, and bilirubin combinations as risk factors for predicting coronary artery disease. Atherosclerosis 2000; 150: 381-7.

8. Celik T, Kaya MG, Akpek M, et al. Does serum bilirubin level on admission predict TIMI flow grade and in-hospital MACE in patients. Angiology 2014; 65: 198-204.

9. Myocardial infarction redefined: a consensus document of The Joint European Society of Cardiology/American College of Cardiology Committee for the redefinition of myocardial infarction. Eur Heart J 2000; 21: 1502-13.

10. Killip T, Kimball JT. Treatment of myocardial infarction in a coronary care unit. A two year experience with 250 patients. Am J Cardiol 1967; 20: 457-64.

11. The Thrombolysis in Myocardial Infarction (TIMI) trial. Phase I findings. TIMI study group. N Engl J Med 1985; 312: 932e6.

12. Gensini GG. A more meaningful scoring system for determining the severity of coronary heart disease. Am J Cardiol 1983; 51: 606.

13. The GUSTO Angiographic investigators. The effects of tissue plasminogen activator, streptokinase, or both on coronary-artery patency, ventricular function, and survival after acute myocardial infarction. N Engl J Med 1993; 329: 1615-22.

14. Acet $\mathrm{H}$, Ertas F, Akıl MA, et al. New inflammatory predictors for non-valvular atrial fibrillation: echocardiographic epicardial fat thickness and neutrophil to lymphocyte ratio. Int J Cardiovasc Imaging 2014; 30: 81-9.

15. Akpek M, Kaya MG, Uyarel $H$, et al. The association of serum uric acid levels on coronary flow in patients with STEMI undergoing primary $\mathrm{PCI}$. Atherosclerosis 2011; 219: 334-41.

16. Wu TW, Wu J, Li RK, et al. Albumin-bound bilirubins protect human ventricular myocytes against oxyradical damage. Biochem Cell Biol 1991; 69: 683-8.

17. Yano Y, Ozono R, Oishi Y, et al. Genetic ablation of the transcription repressor Bach1 leads to myocardial protection against isch emia/reperfusion in mice. Genes Cells 2006; 11: 791-803.
18. Yet SF, Tian R, Layne MD, et al. Cardiac-specific expression of heme oxygenase- 1 protects against ischemia and reperfusion injury in transgenic mice. Circ Res 2001; 89: 168-73.

19. Gullu H, Erdogan D, Tok D, et al. High serum bilirubin concentrations preserve coronary flow reserve and coronary microvascular functions. Arterioscler Thromb Vasc Biol 2005; 25: 2289-94.

20. Erdogan T, Ciçek Y, Kocaman SA, et al. Increased serum bilirubin level is related to good collateral development in patients with chronic total coronary occlusion. Intern Med 2012; 51: 249-55.

21. Gul M, Uyarel H, Ergelen M, et al. Prognostic value of total bilirubin in patients with ST-segment elevation acute myocardial infarction undergoing primary coronary intervention. Am J Cardiol 2013; 111: 166-71.

22. Wei S, Gao C, Wei G, et al. The level of serum bilirubin associated with coronary lesion types in patients with coronary artery disease. J Cardiovasc Med 2012; 13: 432-8.

23. Erkan B, Ekici M, Uğurlu G, et al. The role of bilirubin and its protective function against coronary heart disease. Herz 2013; DOI 10.1007/s00059-013-3872-5. 